\title{
Low levels of high-density lipoprotein cholesterol predict the presence of coronary artery disease in patients with aortic aneurysms
}

Tatsuya Saigusa $^{1}$, MD; Atsushi Izawa ${ }^{1}, \mathrm{MD}, \mathrm{PhD}$; Takashi Miura ${ }^{1}, \mathrm{MD}$; Soichiro Ebisawa ${ }^{1}, \mathrm{MD}_{\text {, }}$ Yuji Shiba ${ }^{1}, \mathrm{MD}, \mathrm{PhD}$; Yusuke Miyashita ${ }^{1}, \mathrm{MD}, \mathrm{PhD}$; Takeshi Tomita ${ }^{1}, \mathrm{MD}, \mathrm{PhD}$; Jun Koyama ${ }^{1}$, MD, PhD; Daisuke Fukui ${ }^{2}$, MD, PhD, Tamaki Takano², MD, PhD, Jun Amano², MD, PhD, Uichi Ikeda $^{1}, \mathrm{MD}, \mathrm{PhD}$

${ }^{1}$ Department of Cardiovascular Medicine, Shinshu University School of Medicine, Matsumoto, Japan

${ }^{2}$ Department of Cardiovascular Surgery, Shinshu University School of Medicine, Matsumoto, Japan Email addresses for all co-authors: TS: tatsuwan@yahoo.co.jp, TM: miuramen@shinshu-u.ac.jp, SE:

ebifender@yahoo.co.jp, YS: yshiba@shinshu-u.ac.jp, YM: ybm1965@shinshu-u.ac.jp, TT: ttomita@shinshu-u.ac.jp, JK: jkoyama@shinshu-u.ac.jp, DF: dfukui@shinshu-u.ac.jp, TT: ttakano@shinshu-u.ac.jp, JA: junamano@shinshu-u.ac.jp, UI: uikeda@shinshu-u.ac.jp.

We have no conflict of interests and grants support with regard to this manuscript.

Total: 2353 words, Abstract: 147 words. Total number of tables: 3, Total number of figures: 1 .

Correspondence to: Atsushi Izawa, $\mathrm{MD}, \mathrm{PhD}$

Department of Cardiovascular Medicine, Shinshu University School of Medicine

3-1-1 Asahi, Matsumoto, Nagano 390-8621, Japan

Tel/Fax: +81-263-37-3352/+81-263-37-2573

E-mail: izawa611@shinshu-u.ac.jp 


\section{Abstract}

To identify predictors of the presence of coronary artery disease (CAD) in patients with planned surgery for aortic aneurysms, we reviewed clinical profiles and angiography records of 191 patients with aortic aneurysms (34 thoracic, 137 abdominal and 20 thoracoabdominal; 162 men; mean age, $75.2 \pm 7.7$ years). The incidence of CAD was $38.7 \%$ among all the patients. Patients with CAD had significantly low levels of high-density lipoprotein cholesterol (HDL-C) as compared with patients without CAD ( $45.5 \pm 9.4$ vs $50.9 \pm 13.4 \mathrm{mg} / \mathrm{dL} ; \mathrm{p}=0.009)$. Multivariate analysis showed that only low HDL-C levels were associated with the presence of CAD (adjusted odds ratio, 0.946; 95\% confidence interval, $0.911-0.983 ; \mathrm{p}=0.004)$. The optimal cut-off level of HDL-C to predict CAD was $47.50 \mathrm{mg} / \mathrm{dL}$. CAD is common in patients with aortic aneurysms and low levels of HDL-C are independently associated with the presence of CAD.

Keywords: Aortic aneurysm $\cdot$ Coronary artery disease $\cdot$ High-density lipoprotein cholesterol 


\section{INTRODUCTION}

Aortic aneurysms may represent systemic atherosclerosis and are associated with common risk factors for cardiovascular disease [1]. A high incidence of coronary artery disease (CAD) has been reported in patients with aortic aneurysms, with the prevalence as high as $30-70 \%[2,3]$. The high comorbidity indicates an etiological association between CAD and abdominal aortic aneurysms (AAA). Large-scale clinical studies to identify perioperative risks for non-cardiac surgery have enabled the development of risk indices/scores [4] that indicate an increase in perioperative cardiovascular events in patients with CAD. Indeed, a previous report has shown that the presence of CAD is associated with late mortality and morbidity after abdominal aortic aneurysmectomy [5]. Thus, preoperative diagnosis of CAD in patients with aortic aneurysms is essential; however, the predictors of comorbid CAD have not been fully investigated. In this study, we examined the incidence and predictors of $\mathrm{CAD}$ in patients with planned surgery for aortic aneurysms.

\section{MATERIALS AND METHODS}

\section{Patients}

The study population comprised 191 patients who were admitted for planned surgery for thoracic aortic aneurysms (TAAs) and/or AAAs between January 2006 and December 2009. Because of the 
high incidence of CAD accompanying aortic aneurysms, coronary angiography was performed in all perioperative patients with aneurysm, regardless of the patient's symptoms, after informed consent was obtained. Patient background details and laboratory examination results on admission were obtained retrospectively from medical records. Hypertension was defined as a systolic blood pressure at rest of $>140 \mathrm{mmHg}$ and/or a diastolic pressure at rest of $>90 \mathrm{mmHg}$ on admission or current treatment with antihypertensive agents. Smoking status was defined as a history of smoking or the smoking of at least 1 cigarette/day at present. Body mass index was calculated and diabetes was evaluated by levels of HbAlc. Patients were excluded if they had been given a previous diagnosis of angiographically significant $\mathrm{CAD}$, history of percutaneous coronary intervention or coronary artery bypass grafting, and/or symptomatic angina. No patients were enrolled without obtaining informed consent for coronary angiography.

\section{Definition of CAD}

Coronary angiography was mostly performed via the radial artery. Diagnoses were made by at least 2 experienced cardiologists. CAD was considered significant in the event of an angiographic luminal stenosis of $\geq 75 \%$ of the epicardial coronary artery or a stenosis of $\geq 50 \%$ of the left main trunk. 


\section{Indication for coronary revascularization}

Procedures for coronary revascularization were performed following the guidelines for elective percutaneous coronary intervention in patients with stable coronary artery disease. The Japanese guidelines are in accordance with those published by the Task Force on Myocardial Revascularization of the European Society of Cardiology and the European Association for Cardio-Thoracic Surgery; European Association for Percutaneous Cardiovascular Interventions [6].

\section{Surgical treatment of aortic aneurysm}

Indications and strategies for surgical intervention for aortic aneurysms were dependent either on the size of aneurysm or the risk of rupture. In general, patients with a TAA of $>6 \mathrm{~cm}$ in diameter or an AAA of $>5 \mathrm{~cm}$ in diameter were recruited. In addition, patients with high-risk aneurysms with a rapid increase in size or that were saccular were also enrolled before surgical intervention. Placement of endovascular stent-grafts was recommended in patients at high risk for open aortic surgery.

Statistical analyses 
Data are presented as mean \pm standard deviation for continuous variables and as numbers and percentages for categorical variables. Continuous variables were compared using the non-paired $t$ test in normal distribution or Mann-Whitney $U$ test in non-normal distribution. Categorical variables were compared using the chi-square test.

Non-paired $t$ tests or the Mann-Whitney $U$ test for continuous data and chi-square test for categorical data were used to compare results between patients with and without CAD. Multivariate logistic regression analysis was used to determine the independent parameters associated with CAD, with adjustment for hypertension, HDL-C, low-density lipoprotein cholesterol (LDL-C), HbA1c, smoking and statin. Odds ratios (OR) and 95\% confidence intervals (CI) were calculated. Factors that were significantly different for patients with and without CAD and common coronary risk factors were entered into multivariate logistic regression analysis. The receiver operating characteristic curve was created to assess diagnostic performance to predict CAD. All statistical analyses were performed using the IBM SPSS Statistics software (ver. 18; IBM, Somers, NY, USA), and results were considered significant when two-tailed $\mathrm{P}$ values were $<0.05$.

\section{RESULTS}

\section{Patient characteristics}


The characteristics of all 191 patients and subgroups by the presence of CAD are shown in Table 1.

The mean level of LDL-C was $112 \pm 28 \mathrm{mg} / \mathrm{dL}$, and other risk factors were practically controlled.

Patients with CAD had low HDL-C levels as compared with those in patients without CAD $(\mathrm{p}=$ 0.009). No other variables showed significant difference between the groups, e.g. hypertension $(\mathrm{p}=$ $0.210)$, LDL-C $(p=0.923)$ and smoking $(p=0.216)$. Without any history of CAD, only $19.4 \%$ patients were treated with statins and those with antiplatelet agents were $21.6 \%$. The characteristics of CAD of all 191 patients and subgroups (34 patients with TAA, 137 with AAA, and 20 with both TAA and AAA) are shown in table 2. The majority was AAA group (71.7\%). Angiographically significant stenoses of coronary arteries were observed in $38.7 \%$ of all patients and in $20.6,40.9$ and $55.0 \%$ of patients with TAA, AAA, and both TAA and AAA, respectively. In all patients with CAD, the involvement of single, double, and triple vessels was 56.8, 28.4 and $14.9 \%$, respectively. Similarly, the main type of CAD was single-vessel disease in each subgroup of patients. These lesions were located in the left anterior descending artery (44.9\%), left circumflex artery (26.0\%), right coronary artery (26.0\%), and left main trunk (3.1\%). Importantly, $54.1 \%$ of patients with significant CAD underwent coronary revascularization before surgical intervention.

Univariate and multiple logistic regression analyses 
Univariate analyses were performed to evaluate factors associated with the presence of CAD in patients with aortic aneurysms. In all patients, low HDL-C levels and the presence of AAA were significantly associated with comorbid CAD $(\mathrm{p}=0.009$ and $\mathrm{p}=0.038$, respectively). Multiple logistic regression analysis indicated that low HDL-C and AAA were independent predictors of CAD (odds ratio [OR], 0.630; 95\% confidence interval [95\%CI], 0.443-0.896; $\mathrm{p}=0.010$ and $\mathrm{OR}$, 3.331; 95\%CI, 1.081-10.261; $\mathrm{p}=0.036$, respectively), with adjustment for hypertension, LDL-C levels, HbA1c levels, smoking and statin (Table 3). We also performed subgroup analysis in patients with TAA, but no independent risk factors were observed after adjustment. In a group of patients with AAA, low HDL-C levels were again correlated with CAD (univariate analysis, $p=0.041$ ), and multiple logistic regression analysis showed that a low HDL-C level was the sole significant predictor of CAD (OR, 0.646; 95\%CI, 0.425-0.981; $\mathrm{p}=0.041)$. Hypertension, LDL-C levels, LDL-C/HDL-C ratio, triglyceride (TG) levels, HbA1c levels, and smoking status were not associated with CAD in the AAA subgroup.

\section{Receiver operating characteristic analysis}

The area under the receiver operating characteristic curve of HDL-C to predict CAD was 0.616 (95\% CI 0.523-0.708, $\mathrm{p}=0.018$ ). The optimal cut-off HDL-C level was $47.50 \mathrm{mg} / \mathrm{dL}$ with the 
sensitivity and specificity of $54.8 \%$ and $62.7 \%$, respectively (Fig. 1). No other variables were applicable for the receiver operating characteristic analysis.

\section{DISCUSSION}

The prevalence of CAD in our group of 191 patients with aortic aneurysms was $38.7 \%$; a low

HDL-C level was an independent and significant predictor of CAD. CAD was diagnosed predominantly as single-vessel disease $(56.8 \%)$, and $54.1 \%$ of the cases with significant CAD required coronary revascularization before surgical intervention for the aneurysm. In general, the prevalence of CAD is 8.13-11.8 per 1000 in the Japanese population [7]; accordingly, we observed a significantly high incidence of CAD in patients with aortic aneurysms.

Several guidelines state that atherosclerosis can be a common background for CAD and AAA [8, 9], and the presence of $\mathrm{CAD}$ is associated with higher perioperative risk for AAA patients [10]. In addition, AAAs can be considered as CAD equivalent based on our results. Compared to the incidence of CAD in patients with AAAs, a relatively low incidence of CAD (20.6\%) was observed in patients with TAAs. This can be explained in part by the unique mechanism of TAA formation, which has been identified as a remodeling process of the extracellular matrix, e.g. dysregulation of transforming growth factor-beta (TGF-beta) signaling [11]. The highest prevalence of CAD was 
observed in patients with both TAA and AAA (55.0\%); however, confirmation of this association and description of the detailed pathophysiology warrants a study with a large number of patients.

Previous studies have shown that low HDL-C levels are associated with the presence of CAD independently of the risk estimated using a high LDL-C level [12, 13]. To our knowledge, this is the first study showing the sole association of CAD with low HDL-C levels in patients with aortic aneurysms. The association between low HDL-C levels and advanced cardiovascular risk has been highlighted in patients treated with statin in whom serum LDL-C levels were maintained at $<70$ $\mathrm{mg} / \mathrm{dL}$, in a post hoc analysis of the TNT trial [14]. The METEOR substudy showed that low HDL-C levels are associated with echolucent plaques of the carotid intima-media, suggesting a high risk of cardiovascular events [15]. These studies clearly highlight the critical role of HDL-C in preventing the progression of atherosclerosis. A recent meta-analysis has demonstrated that treatment with statin therapy does not alter the association between low levels of HDL-C and increased cardiovascular risk [16]. Current guidelines recommend statin use in patients undergoing coronary artery bypass grafting [17]; however, beneficial effects in non-cardiac vascular surgery patients have not been fully elucidated [18]. Although we did not have enough data to show statin effect in patients with aortic aneurysms, we may recommend perioperative statin use as shown in a recent study [19]. 
The critical importance of HDL-C function as well as HDL-C levels can be explained by HDL-C-mediated efflux of cholesterol from the peripheral arterial wall to the liver, i.e. reverse cholesterol transport [20]. This transport has been thought to prevent atherosclerosis in the following ways: macrophage-specific or non-specific arterial wall efflux, non-arterial wall efflux, lipoprotein transport, hepatic cholesterol uptake, and direct excretion into small intestine or fecal excretion [21]. Efflux capacity was a strong inverse predictor of coronary disease status, independent of the HDL-C level [22]. In addition, decreased cholesterol efflux has been suggested to be a residual risk factor in statin-treated patients with a low HDL-C level [23]. Thus, not only low serum levels, but the dysfunction of HDL-C [24] can be a new target to treat patients with cardiometabolic risk factors and/or atherosclerosis. Further research is required to examine HDL-C function in patients with aortic aneurysms.

Peripheral arterial disease (PAD) is a manifestation of systemic atherosclerosis [3] and is an important predictor of concomitant atherosclerotic diseases [25]. The Reduction of Atherothrombosis for Continued Health (REACH) registry, a worldwide cohort study, showed that almost $30 \%$ of patients with PAD also have CAD [26]. Recent reports have also indicated that PAD is significantly associated with CAD in patients with AAA [27] and that myocardial ischemia is more frequent in patients with combined PAD and an aortic aneurysm (73\%) than in those with 
PAD (55\%) only or those with an aortic aneurysm (37\%) only [25]. In the current study, however, no correlation was observed between the presence of CAD and the ankle-brachial pressure index, a marker of PAD, probably because of this specific population of patients with aortic aneurysms. No other predictors were identified in our study, which included aspects of patient's medical histories and treatment (e.g. statins and antiplatelet agents).

Recent reports have indicated that non-fasting TG levels [28] as well as TG/HDL-C ratios and non-HDL-C levels [29] are also independently correlated with cardiovascular events. In our study, however, the statistically significant factor was a low HDL-C level alone and no other lipid profile parameter was associated with comorbid CAD with aortic aneurysm. Interestingly, HDL-C has been identified as the most important lipid variable for predicting the risk of AAA development [8, 30]. Low HDL-C levels, therefore, appear to play an important role in the development of AAA as well as in the comorbidity CAD of these patients. Thus, lipid-modifying strategies should be further focused on raising HDL-C levels to reduce cardiovascular events. The clinical benefits of an increase in HDL-C levels have been highlighted in the Framingham Heart Study, where an increase in HDL-C by $1 \mathrm{mg} / \mathrm{dL}$ resulted in a $2-3 \%$ decrease in the incidence of CAD [31]. Mabuchi et al. revealed that a $10 \mathrm{mg} / \mathrm{dL}$ increase in HDL-C lowered the relative risk of a coronary event by $28.3 \%$ [32]. Our study indicated that the reduction in the relative risk of CAD with additional $1 \mathrm{mg} / \mathrm{dL}$ 
decrease in the HDL-C level was $6.3 \%$.

\section{Study limitations}

First, a potential selection bias can be expected in this small cohort study. Second, CAD was angiographically defined and was not identical to actual myocardial ischemia with any findings on myocardial perfusion images. Third, the results of this study may predominantly reflect data from the AAA group, because of the predominant number of patients with AAA. Fourth, the rationale for low HDL-C levels being the sole predictor for CAD in this study is unknown, although common risk factors play important roles in the development of cardiovascular disease. Fifth, HDL-C function and cholesterol efflux capacity were not evaluated. Further investigations in large-scale prospective clinical studies and/or basic sciences are necessary to elucidate the roles of HDL-C and its function in the pathophysiological association between CAD and aortic aneurysms.

Although routine preoperative coronary angiography is not strongly recommended for patients with few cardiovascular risks and with functional exertion capacity of $>4$ metabolic equivalents [33], this study and previous studies consistently show a high prevalence of CAD especially in patients with AAA. Accordingly, we encourage clinicians to evaluate CAD intensively in patients with low HDL-C levels, before surgical intervention for aortic aneurysms. 


\section{CONCLUSIONS}

A low HDL-C level was an independent predictor of comorbid CAD in patients with aortic aneurysms. A high prevalence of CAD in patients with aortic aneurysms highlights the importance of surgical risk stratification according to HDL-C levels to identify patients with comorbid CAD.

Conflict of interest: none declared. 


\section{Tables}

Table 1

Clinical characteristics

\begin{tabular}{|c|c|c|c|c|}
\hline & $\begin{array}{c}\text { All } \\
n=191\end{array}$ & $\begin{array}{l}\text { CAD (-) } \\
n=116\end{array}$ & $\begin{array}{c}\mathrm{CAD}(+) \\
n=75\end{array}$ & p value \\
\hline \multicolumn{5}{|l|}{ Clinical } \\
\hline Age (yrs) & $75.2 \pm 7.7$ & $74.8 \pm 7.8$ & $75.9 \pm 7.5$ & 0.316 \\
\hline Male & $162(84.8 \%)$ & $97(83.6 \%)$ & $65(86.7 \%)$ & 0.567 \\
\hline $\mathrm{BMI}\left(\mathrm{kg} / \mathrm{m}^{2}\right)$ & $22.9 \pm 3.3$ & $23.0 \pm 3.3$ & $22.9 \pm 3.4$ & 0.977 \\
\hline TAA diameter $(\mathrm{mm})$ & $52.9 \pm 10.6$ & $51.6 \pm 11.6$ & $55.7 \pm 7.7$ & 0.221 \\
\hline AAA diameter $(\mathrm{mm})$ & $51.5 \pm 11.2$ & $50.4 \pm 11.2$ & $52.8 \pm 11.2$ & 0.235 \\
\hline Illiac artery aneurysm & $56(29.6 \%)$ & $31(27.0 \%)$ & $25(33.8 \%)$ & 0.316 \\
\hline $\mathrm{ABI}<0.9$ & $23(15.7 \%)$ & $13(15.1 \%)$ & $10(16.7 \%)$ & 0.8 \\
\hline Hypertension & $129(75.0 \%)$ & $73(71.6 \%)$ & $56(80.0 \%)$ & 0.21 \\
\hline Smoking & $103(63.9 \%)$ & $67(67.7 \%)$ & $36(58.1 \%)$ & 0.216 \\
\hline \multicolumn{5}{|l|}{ Laboratory } \\
\hline HbA1c (\%) & $5.5 \pm 0.7$ & $5.5 \pm 0.8$ & $5.5 \pm 0.5$ & 0.776 \\
\hline LDL-C (mg/dL) & $112 \pm 28$ & $112 \pm 27$ & $112 \pm 29$ & 0.923 \\
\hline $\mathrm{HDL}-\mathrm{C}(\mathrm{mg} / \mathrm{dL})$ & $49 \pm 12$ & $51 \pm 13$ & $46 \pm 9$ & 0.009 \\
\hline $\mathrm{LDL}-\mathrm{C} / \mathrm{HDL}-\mathrm{C}$ & $2.4 \pm 0.9$ & $2.4 \pm 0.8$ & $2.5 \pm 1.0$ & 0.49 \\
\hline Triglyceride (mg/dL) & $135 \pm 75$ & $125 \pm 56$ & $149 \pm 95$ & 0.197 \\
\hline $\mathrm{BNP}(\mathrm{pg} / \mathrm{mL})$ & $66.0 \pm 84.8$ & $64.4 \pm 87.2$ & $68.4 \pm 81.9$ & 0.938 \\
\hline FDP-DD $(\mu \mathrm{g} / \mathrm{mL})$ & $6.0 \pm 7.2$ & $5.9 \pm 6.3$ & $6.2 \pm 8.3$ & 0.787 \\
\hline \multicolumn{5}{|l|}{ Medication } \\
\hline Statin & $37(19.4 \%)$ & $16(13.8 \%)$ & $21(28.0 \%)$ & 0.015 \\
\hline Antiplatelet & $41(21.6 \%)$ & $20(17.2 \%)$ & $21(28.4 \%)$ & 0.069 \\
\hline
\end{tabular}

Hypertension was defined as systolic blood pressure at rest of $>140 \mathrm{mmHg}$, and/or diastolic pressure at rest of $>90 \mathrm{mmHg}$ on admission or current treatment with antihypertensive agents.

TAA, thoracic aortic aneurysm; AAA, abdominal aortic aneurysm; BMI, body mass index; $\mathrm{ABI}$, ankle brachial pressure index; LDL-C, low-density lipoprotein cholesterol; HDL-C, high-density lipoprotein cholesterol; BNP, brain natriuretic peptide; FDP-DD, fibrin/fibrinogen degradation products-D dimmer. 
Table 2

Characteristics of coronary artery disease

\begin{tabular}{lcccc}
\hline & All & \multicolumn{3}{c}{ Aneurysm type } \\
\cline { 3 - 5 } & $n=191$ & TAA & AAA & TAA+AAA \\
& $74(38.7)$ & $7(20.6)$ & $56(40.9)$ & $11(55.0)$ \\
Coronary artery disease & & & & \\
Diseased vessel & & & & \\
Single & $42(56.8)$ & $5(71.4)$ & $31(55.4)$ & $6(54.4)$ \\
Double & $21(28.4)$ & $1(14.3)$ & $15(26.8)$ & $5(45.5)$ \\
Triple & $11(14.9)$ & $1(14.3)$ & $10(17.9)$ & 0 \\
Branch location & & & & \\
LAD & $57(44.9)$ & $6(54.4)$ & $46(48.9)$ & $5(27.8)$ \\
LCX & $33(26.0)$ & $3(27.3)$ & $25(26.6)$ & $5(27.8)$ \\
RCA & $33(26.0)$ & $2(18.2)$ & $23(24.5)$ & $8(44.4)$ \\
LMT & $4(3.1)$ & 0 & $4(4.3)$ & 0 \\
Revascularization & $40(54.1)$ & $5(71.4)$ & $28(50.0)$ & $7(63.6)$ \\
\hline
\end{tabular}

Values are $n(\%)$.

AAA, abdominal aortic aneurysm; TAA, thoracic aortic aneurysm.

LAD, left anterior descending artery; LCX, left circumflex artery; RCA, right coronary artery; LMT, left main trunk artery. 
Table 3

Logistic regression analyses for predictors of coronary artery disease

\begin{tabular}{|c|c|c|c|c|c|c|c|c|}
\hline & \multicolumn{4}{|c|}{ Continuous } & \multicolumn{4}{|c|}{ Quartile } \\
\hline & Univariate $p$ value & Multivariate $\mathrm{p}$ value ${ }^{*}$ & OR & $95 \% \mathrm{Cl}$ & Univariate $p$ value & Multivariate $p$ value ${ }^{*}$ & OR & $95 \% \mathrm{Cl}$ \\
\hline Age & 0.217 & & & & 0.162 & 0.304 & & \\
\hline Male & 0.567 & & & & & & & \\
\hline Hypertension & 0.21 & & & & & & & \\
\hline LDL-C & 0.923 & & & & 0.738 & & & \\
\hline HDL-C & 0.009 & 0.004 & 0.946 & $0.911-0.983$ & 0.041 & 0.01 & 0.63 & $0.443-0.896$ \\
\hline LDL-C/HDL-C & 0.49 & & & & 0.795 & & & \\
\hline Triglyceride & 0.077 & 0.269 & & & 0.185 & 0.506 & & \\
\hline Triglyceride/HDL-C & 0.141 & 0.221 & & & 0.113 & 0.353 & & \\
\hline $\mathrm{HbA} 1 \mathrm{c}$ & 0.592 & & & & 0.686 & & & \\
\hline Smoking & 0.216 & & & & & & & \\
\hline TAA & 0.468 & & & & & & & \\
\hline AAA & 0.038 & 0.036 & 3.331 & $1.081-10.261$ & & & & \\
\hline
\end{tabular}

•Adjustment for hypertension, HDL-cholesterol, LDL-cholesterol, HbA1c, smoking and statin.

AAA, abdominal aortic aneurysm; TAA, thoracic aortic aneurysm; OR, odds ratio; $\mathrm{Cl}$, confidence interval.

\section{Figure legends}

Figure 1: Receiver operating characteristic curve of HDL-C to predict coronary artery disease. The area under the curve was $0.616(95 \% \mathrm{CI} 0.523-0.708, \mathrm{p}=0.018)$. The optimal cut-off HDL-C level was $47.50 \mathrm{mg} / \mathrm{dL}$ with $54.8 \%$ sensitivity and $62.7 \%$ specificity. 


\section{REFERENCES}

[1] Takigawa M, Yoshimuta T, Akutsu K, Takeshita S, Yokoyama N. Prevalence and predictors of coexistent silent atherosclerotic cardiovascular disease in patients with abdominal aortic aneurysm without previous symptomatic cardiovascular diseases. Angiology. 2012;63(5):380-385.

[2] Svensjo S, Bjorck M, Gurtelschmid M, Djavani Gidlund K, Hellberg A, Wanhainen A. Low prevalence of abdominal aortic aneurysm among 65-year-old Swedish men indicates a change in the epidemiology of the disease. Circulation. 2011;124(10):1118-1123.

[3] Sukhija R, Aronow WS, Yalamanchili K, Sinha N, Babu S. Prevalence of coronary artery disease, lower extremity peripheral arterial disease, and cerebrovascular disease in 110 men with an abdominal aortic aneurysm. Am J Cardiol. 2004;94(10):1358-1359.

[4] Gupta PK, Gupta H, Sundaram A, Kaushik M, Fang X, Miller WJ et al. Development and validation of a risk calculator for prediction of cardiac risk after surgery. Circulation. 2011;124(4):381-387.

[5] Roger VL, Ballard DJ, Hallett JW, Jr., Osmundson PJ, Puetz PA, Gersh BJ. Influence of coronary artery disease on morbidity and mortality after abdominal aortic aneurysmectomy: a population-based study, 1971-1987. JAm Coll Cardiol. 1989;14(5):1245-1252.

[6] Kolh P, Wijns W, Danchin N, Di Mario C, Falk V, Folliguet T et al. Guidelines on myocardial revascularization. Eur J Cardiothorac Surg. 2010;38 Suppl:S1-S52.

[7] Kiyohara Y. The Hisayama Study. Nihon Rinsho. 2010;68(5):853-856.

[8] Nordon IM, Hinchliffe RJ, Loftus IM, Thompson MM. Pathophysiology and epidemiology of abdominal aortic aneurysms. Nat Rev Cardiol. 2011;8(2):92-102.

[9] Hertzer NR, Beven EG, Young JR, O'Hara PJ, Ruschhaupt WF, 3rd, Graor RA et al. Coronary artery disease in peripheral vascular patients. A classification of 1000 coronary angiograms and results of surgical management. Ann Surg. 1984;199(2):223-233.

[10] Fleisher LA, Beckman JA, Brown KA, Calkins H, Chaikof E, Fleischmann KE et al. ACC/AHA 2007 guidelines on perioperative cardiovascular evaluation and care for noncardiac surgery: a report of the American College of Cardiology/American Heart Association Task Force on Practice Guidelines (Writing Committee to Revise the 2002 Guidelines on Perioperative Cardiovascular Evaluation for 
Noncardiac Surgery): developed in collaboration with the American Society of Echocardiography, American Society of Nuclear Cardiology, Heart Rhythm Society, Society of Cardiovascular Anesthesiologists, Society for Cardiovascular Angiography and Interventions, Society for Vascular Medicine and Biology, and Society for Vascular Surgery. Circulation. 2007;116(17):e418-499.

[11] J E. Medionecrosis aortae idiopathica cystica. Virchows Arch Path Anat. 1930;276:187.

[12] Gordon DJ, Rifkind BM. High-density lipoprotein--the clinical implications of recent studies. $N$ Engl J Med. 1989;321(19):1311-1316.

[13] Goldbourt U, Yaari S, Medalie JH. Isolated low HDL cholesterol as a risk factor for coronary heart disease mortality. A 21-year follow-up of 8000 men. Arterioscler Thromb Vasc Biol. 1997;17(1):107-113.

[14] Arsenault BJ, Barter P, DeMicco DA, Bao W, Preston GM, LaRosa JC et al. Prediction of cardiovascular events in statin-treated stable coronary patients by lipid and nonlipid biomarkers. $J A m$ Coll Cardiol. 2011;57(1):63-69.

[15] Peters SA, Lind L, Palmer MK, Grobbee DE, Crouse JR, 3rd, O'Leary DH et al. Increased age, high body mass index and low HDL-C levels are related to an echolucent carotid intima-media: the METEOR study. J Intern Med. 2011.

[16] Jafri H, Alsheikh-Ali AA, Karas RH. Meta-analysis: statin therapy does not alter the association between low levels of high-density lipoprotein cholesterol and increased cardiovascular risk. Ann Intern Med. 2010;153(12):800-808.

[17] Kuhn EW, Liakopoulos OJ, Choi YH, Wahlers T. Current evidence for perioperative statins in cardiac surgery. Ann Thorac Surg. 2011;92(1):372-379.

[18] Stalenhoef AF. The benefit of statins in non-cardiac vascular surgery patients. $J$ Vasc Surg. 2009;49(1):260-265.

[19] Paraskevas KI, Wierzbicki AS, Mikhailidis DP. Statins and noncardiac vascular disease. Curr Opin Cardiol. 2012;27(4):392-397.

[20] Glomset JA. The metabolic role of lecithin: cholesterol acyltransferase: perspectives from pathology. Adv Lipid Res. 1973;11(0):1-65.

[21] Rosenson RS, Brewer HB, Jr., Davidson WS, Fayad ZA, Fuster V, Goldstein J et al. Cholesterol efflux and atheroprotection: advancing the concept of reverse cholesterol transport. Circulation. 
2012;125(15):1905-1919.

[22] Khera AV, Cuchel M, de la Llera-Moya M, Rodrigues A, Burke MF, Jafri K et al. Cholesterol efflux capacity, high-density lipoprotein function, and atherosclerosis. $N$ Engl J Med. 2011;364(2):127-135.

[23] Posadas-Sanchez R, Posadas-Romero C, Mendoza-Perez E, Caracas-Portilla NA, Cardoso-Saldana G, Medina-Urrutia A et al. Cholesterol efflux and metabolic abnormalities associated with low high-density-lipoprotein-cholesterol and high triglycerides in statin-treated coronary men with low-density lipoprotein-cholesterol <70 mg/dl. Am J Cardiol. 2012;109(5):636-641.

[24] Otocka-Kmiecik A, Mikhailidis DP, Nicholls SJ, Davidson M, Rysz J, Banach M. Dysfunctional HDL: a novel important diagnostic and therapeutic target in cardiovascular disease? Prog Lipid Res. 2012;51(4):314-324.

[25] Hirose K, Chikamori T, Hida S, Tanaka H, Igarashi Y, Watanabe Y et al. Prevalence of coronary heart disease in patients with aortic aneurysm and/or peripheral artery disease. Am J Cardiol. 2009;103(9):1215-1220.

[26] Steg PG, Bhatt DL, Wilson PW, D'Agostino R, Sr., Ohman EM, Rother J et al. One-year cardiovascular event rates in outpatients with atherothrombosis. Jama. 2007;297(11):1197-1206. [27] Liu Y, Luo JF, Huang WH, Wang HY, Xie NJ, Fan RX et al. Incidence and risk factors of coronary artery disease in elderly patients with abdominal aortic aneurysm. Zhonghua Xin Xue Guan Bing Za Zhi. 2011;39(1):53-56.

[28] Mora S, Rifai N, Buring JE, Ridker PM. Fasting compared with nonfasting lipids and apolipoproteins for predicting incident cardiovascular events. Circulation. 2008;118(10):993-1001. [29] Pischon T, Girman CJ, Sacks FM, Rifai N, Stampfer MJ, Rimm EB. Non-high-density lipoprotein cholesterol and apolipoprotein B in the prediction of coronary heart disease in men. Circulation. 2005;112(22):3375-3383.

[30] Golledge J, van Bockxmeer F, Jamrozik K, McCann M, Norman PE. Association between serum lipoproteins and abdominal aortic aneurysm. Am J Cardiol. 2010;105(10):1480-1484.

[31] Gordon T, Castelli WP, Hjortland MC, Kannel WB, Dawber TR. High density lipoprotein as a protective factor against coronary heart disease. The Framingham Study. Am J Med. 1977;62(5):707-714. 
[32] Mabuchi H, Kita T, Matsuzaki M, Matsuzawa Y, Nakaya N, Oikawa S et al. Large scale cohort study of the relationship between serum cholesterol concentration and coronary events with low-dose simvastatin therapy in Japanese patients with hypercholesterolemia and coronary heart disease: secondary prevention cohort study of the Japan Lipid Intervention Trial (J-LIT). Circ J. 2002;66(12):1096-1100.

[33] Eagle KA, Berger PB, Calkins H, Chaitman BR, Ewy GA, Fleischmann KE et al. ACC/AHA guideline update for perioperative cardiovascular evaluation for noncardiac surgery---executive summary a report of the American College of Cardiology/American Heart Association Task Force on Practice Guidelines (Committee to Update the 1996 Guidelines on Perioperative Cardiovascular Evaluation for Noncardiac Surgery). Circulation. 2002;105(10):1257-1267. 